\title{
On Cheating and Whistle-Blowing
}

Aleksander Berentsen

Esther Brügger

Simon Lörtscher

03-02

April 2003

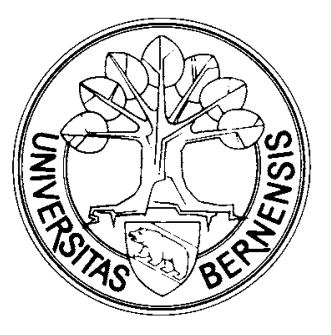

Universität Bern

Volkswirtschaftliches Institut

Gesellschaftstrasse 49

3012 Bern, Switzerland

Tel: 41 (0)31 6314506

Web: www.vwi.unibe.ch 


\title{
On Cheating and Whistle-Blowing*
}

\author{
Aleksander Berentsen, Esther Brügger and Simon Lörtscher ${ }^{\dagger}$
}

April 14, 2003

\begin{abstract}
We study the role of whistle-blowing in the following inspection game. Two agents who compete for a valuable prize can either behave legally or illegally. After the competition, a controller investigates the agents' behavior. This control game has a unique equilibrium in mixed strategies. We then add a whistle-blowing stage, where the controller asks the loser to blow the whistle. This extended game has a unique perfect Bayesian equilibrium in which only a cheating loser accuses the winner of cheating and the controller tests the winner if and only if the winner is accused of cheating. Whistle-blowing reduces the frequencies of cheating, is less costly in terms of test frequencies, and leads to a strict Pareto-improvement if punishments for cheating are sufficiently large.
\end{abstract}

KEYWORDS: Principal-two-Agents, Inspection Games, Asymmetric Information, Signalling.

JEL-Classification: C72, D82, K42.

${ }^{*}$ We would like to thank Ben Johnson ;-) for inspiration. This paper has also benefited from the comments of seminar participants at the University of Bern, and conference participants at the SSES-meeting in Bern. The second and third author gratefully acknowledge financial support from a grant received by the WWZ-Forum Basel.

${ }^{\dagger}$ Berentsen: Economics Department, University of Basel, Petersgraben 51, CH4003 Basel. E-mail: aleksander.berentsen@unibas.ch Brügger: Economics Department, University of Bern, Gesellschaftsstrasse 49, CH-3012 Bern. E-mail: esther.bruegger@vwi.unibe.ch Lörtscher: Economics Department, University of Bern, Vereinsweg 23, CH-3012 Bern. E-mail: simon.loertscher@vwi.unibe.ch 


\section{Introduction}

We address the following problem. Two agents who compete for a prize can choose between two strategies: behave legally or illegally. If both agents play the same strategy, either agent wins the prize with an exogenously given probability. If only one player plays the illegal strategy ("cheats"), he wins with certainty. According to these assumptions, cheating is a dominant strategy. This calls for an outside authority - the controller - to control agents' behavior, provided there is social preference against cheating. The controller faces the problem that the agents' behavior is not publicly observable such that detecting cheating imposes costs. Even if it pays to control an agent's behavior if there is a high probability that he cheats, it will not pay to control an agent if he is known to play legally. Agents, on the other hand, are inclined not to cheat if they know that they will be controlled, while they have incentives to cheat if they know that there will be no controls. We refer to such a game as a control problem. In general, a control problem has an equilibrium in mixed strategies only.

There are many real world situations that have this control problem property. An obvious example is doping in sports, where a player can increase his prospects to prevail over his opponent by doping. Another example is the enforcement of environmental law. Any firm can increase its profits by not using the appropriate, but expensive abatement technology if the firm can be sure not be detected. Tax avoidance is another example (Greenberg [8]). The control problem also applies for firms who compete for a valuable resource like, say, capital. In order to attract capital, firms can either truthfully report accounting and other data, or they can cheat. The practice of cheating is not uncommon. According to the Economist magazine [6] some company's "sales forecasts were so high that managers, who were meant to use them to plan production, routinely ignored the numbers."

In the unique equilibrium of the control game, both agents randomize between cheating and not cheating. Therefore, the strategy an agent chooses in equilibrium is a probability distribution over his pure strategies, deter- 
mined by the parameters of the game. This equilibrium strategy of an agent has to be distinguished from the actual behavior of the agent in equilibrium, which is the realization of a random variable. It is either "cheat" or "not cheat". While all rational agents infer the equilibrium distributions, we assume that the realizations of the random variables are only privately observed. That is, whether an agent actually cheats or not is only observed by himself. Given the assumption that cheating is powerful in the sense that a cheater always prevails over a player who does not cheat, a player for whom the realization of his random variable was "cheat" and who loses the contest can therefore infer that the winner must have cheated, too. Our goal is then to give the controller the possibility to make use of this private information. We model this by adding an additional stage to the control game, which we call whistle-blowing stage. In this stage, the loser is given the opportunity to hand out to the controller additional information the loser might have. The problem then consists of designing an incentive-compatible remuneration scheme, which induces the loser to send different messages to the controller depending on whether he has cheated or not. We show under what conditions such a scheme exists. If it exists, it always reduces the frequency of tests and if the penalty for cheating is sufficiently large, it also reduces the frequency of cheating. We also show that whistle-blowing leads to a strict Pareto-improvement if the penalty is sufficiently large.

The game between the two competing agents and the controller is similar to a standard principal-agent problem. There is, however, an important difference between the two. In a standard principal-agent setting, greater effort increases an agent's chances of winning and the principal's welfare. In our model, the cheating strategy that increases the prospect of winning is disliked by society. Therefore, the illegal activity (cheating or doping) is a dominant strategy in the absence of controls. Our paper is also related to the literature on inspection games (an overview on such games is given by Avenhaus et al. [3]). In contrast to a standard inspection game, the inspected agents are competitors. This is not the case in the inspection games we are aware of, where typically there is but one inspector and one 
inspectee (see e.g. Güth and Pethig [9]). On the other hand, we do not address statistical test problems that play a prevalent role in the paper by Avenhaus et al. [3]. An obvious application of inspection games are arms inspection and disarmament, which are often based on (e.g. nuclear) material accountancy. Whether the competitive side of a problem can be neglected surely depends on the question addressed. Competition may be less important in arms inspection than it is in, say, sports, though one can easily think of counter-examples. Finally, our approach is also related to the literature on tournaments and contests, which started with Lazear and Rosen [11] (see also Dixit [5]). While this literature typically addresses the question under what conditions tournaments are optimal remuneration schemes, our starting point is different insofar as we take the contest as given and rather investigate how contestants behave under different monitoring schemes.

The paper is structured as follows. In Section 2 we introduce the control problem. Section 3 investigates the whistle-blowing game, and Section 4 compares the two mechanisms. Section 5 concludes.

\section{The Control Problem}

We first consider what we call the control problem. A control problem describes a situation where one or more players - to which we will refer as agents - can choose between behaving legally and illegally, while another player - called the controller - can choose between controlling (or testing) and not controlling the agent(s). Preferences in control problems are such that agents prefer to behave illegally (or to "cheat") if the controller does not control, while they prefer to behave legally if there is control. The controller, on the other hand, prefers not to control if agents play legally, while if they play cheat, he prefers to control. In general, therefore, control problems are games with no equilibrium in pure strategies. ${ }^{1}$

\footnotetext{
${ }^{1}$ An example for a typical control problem is fare dodging in public transport (Avenhaus [2]). Provided there are no fare dodgers, it makes no sense for the provider of the transport facilities to make costly controls. But provided there are no controls, it makes no sense
} 
Unlike in standard control problems with one agent, where the agent's payoff is directly affected only by his own and the principal's choice of strategies, we study a situation in which two agents compete for a valuable resource (a prize) only one of them can win. Cheating increases an agent's chances of winning the competition. Thus, an agent's payoff is not only affected by his own choice of strategy and that of the principal or controller, but also by the strategy chosen by the other agent.

We describe the control problem in the context of sports competition, where the agents are two athletes who face the choice of playing "clean" or playing "doped". Society's objective is to reduce the frequency of testing and the frequency of doping. ${ }^{2}$ Given the examples and illustrations discussed in the Introduction, adaptation of the game to other settings with similar structures is straightforward.

\subsection{The Game}

The prize the agents compete for has value 1 for either agent. The a priori probability that agent 1 wins the prize if both agents are clean or if both agents are doped is $\sigma>\frac{1}{2}$. The probability that agent 2 wins is accordingly $1-\sigma$, so that agent 1 is the better player. ${ }^{3}$ The winning probabilities are exogenous to the game. We assume that doping is completely effective in the sense that any doped agent prevails with certainty over his opponent if the opponent is clean. These probabilities are common knowledge.

After the competition, the controller decides whether to test the winner. The controller's set of pure strategies is $\{T, N T\}$, where $T$ stands for test and $N T$ for no test. Consistent with the assumption that the a priori probability

to pay the fare for the user of the transport facility, and provided no fares are paid, controls make perfectly sense. Thus, there is no equilibrium in pure strategies. Customers will cheat with a positive probability smaller than one, and controls will be made with a positive probability, which is also smaller than one.

${ }^{2}$ For a game theoretic analysis of doping and the fight against doping see Berentsen $[4]$.

${ }^{3}$ In sports, for example, $\sigma$ is the probability that 1 prevails over 2 in a pairwise meeting. If it were to be determined in practice, the odds set by bookmakers could be used to approximate $\sigma$. 
$\sigma$ is common knowledge, we assume that the controller also knows which agent is the better one. Therefore, the controller can use different testing probabilities for either agent. The probability that agent 1 is tested if he wins is denoted by $t_{1}$ and the probability that agent 2 is tested after winning is $t_{2}$. Thus, $t_{1}$ and $t_{2}$ denote the controller's mixed strategy of testing winner 1 and winner 2, respectively. The controller's payoffs are such that testing causes him costs of $k \in(0,1)$. The test is reliable, i.e. there are no test errors. If the test indicates that the winner is doped, the controller receives a payoff of $1-k>0$, while if the test indicates that the winner is clean, the controller gets $0-k<0$. $^{4}$

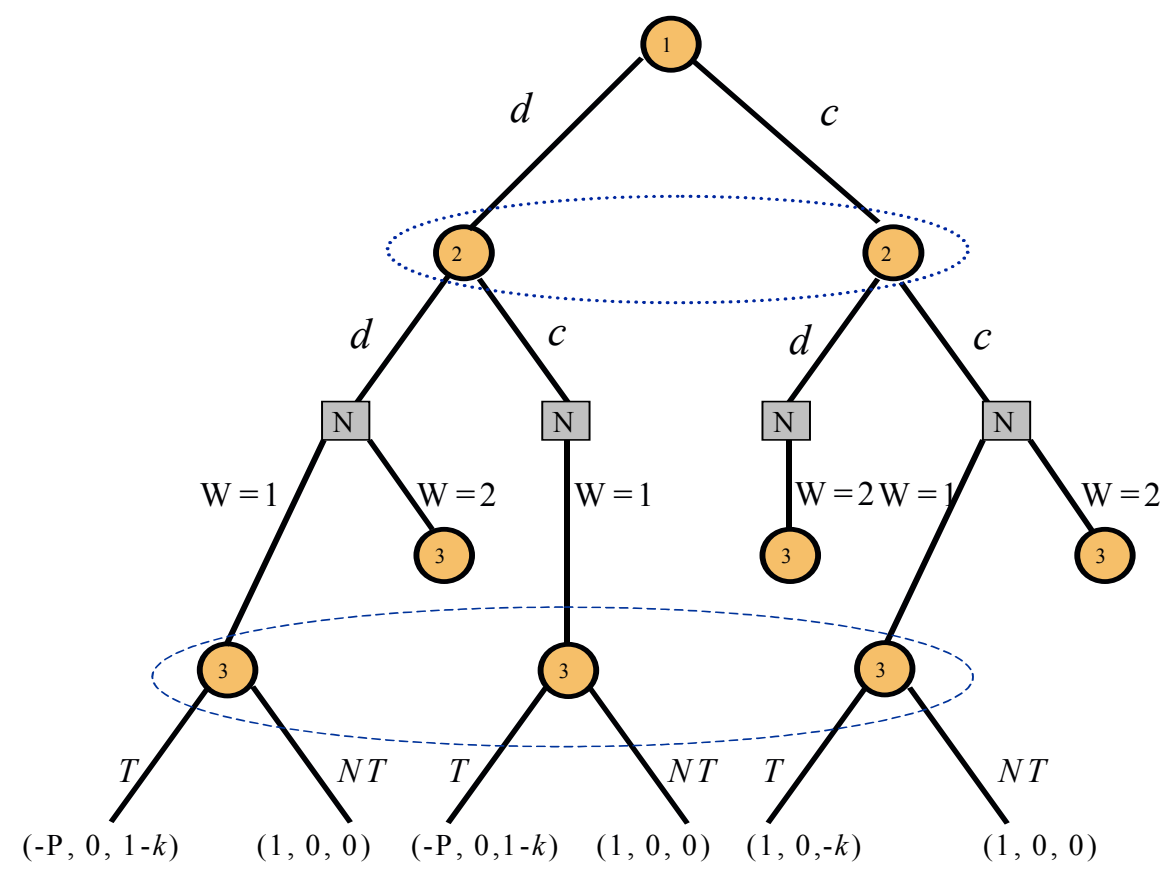

Figure 1: The control problem.

Our control problem is depicted in Figure 1. In order to keep the figure simple, we have not drawn the information set of the controller when agent 1 is the winner.

\footnotetext{
${ }^{4}$ One can also consider the control problem as a game between three players, where player three - the controller - also competes for the prize. The controller wins the prize if he proves that the winner of the contest has cheated. In this setting the controller would never test the loser because his payoff would be $-k$.
} 
Agents' sets of pure strategies are $\{c, d\}$. The probability that agent 1 dopes is denoted by $\alpha$ and the probability that agent 2 dopes is $\beta$. We denote by $X(\alpha)$ the random variable for agent 1 if he dopes with probability $\alpha$. Accordingly, $X(\beta)$ denotes the random variable for agent 2 .

If a player after winning is tested positive, he does not receive the prize and gets the punishment $P \geq 0 .{ }^{5}$ The loser's payoff is 0 , regardless of whether or not he has doped. The punishment $P$ leaves the payoffs of the controller unaffected. Such deadweight loss penalties are an accurate way of modelling, provided the penalty consists of disqualification and a ban from further competitions of detected cheaters, which is the case in sports for athletes who are convicted as dopers. It is also accurate for cheaters within hierarchical contests where detected cheaters are fired or sent to jail or in arms inspection games, provided the arms inspectors find are of no use to themselves. ${ }^{6}$ Finally, throughout the paper we assume that in contrast to the testing probabilities punishments and rewards cannot be conditioned on individual agents. This represents the idea of equality before the law.

\subsection{Equilibrium}

Before we investigate the equilibrium, it is useful to write down the a priori probabilities for either agent to win the game, and the conditional probabilities for agent 1 and 2 to be doped, conditional on winning the game. Let $P(W=1)$ and $P(W=2)$ denote the a priori probabilities that the winner is agent 1 or agent 2 , respectively. These probabilities are

$$
\begin{aligned}
& P(W=1)=\sigma(1-\alpha)(1-\beta)+\alpha(1-\beta)+\sigma \alpha \beta \text { and } \\
& P(W=2)=(1-\sigma)(1-\alpha)(1-\beta)+\beta(1-\alpha)+(1-\sigma) \alpha \beta .
\end{aligned}
$$

\footnotetext{
${ }^{5}$ If $P=0$, the punishment is disqualification so that the winner does not receive the prize. $P>0$ implies that the principal can enforce some additional punishment in terms of utility to a cheater.

${ }^{6}$ Deadweight loss penalties are inaccurate for situations where the penalty is a transfer from the cheating agent to the controller, which is for example the case for fare dodgers (as in Avenhaus [2]). It is straightforward to change our framework to cover situations where the penalty is a transfer from the cheating agent to the controller.
} 
The term $\sigma(1-\alpha)(1-\beta)$ is the probability that player 1 wins times the probabilities that both players are clean. The term $\alpha(1-\beta)$ is the probability that only player 1 cheats in which case he wins with certainty and the term $\sigma \alpha \beta$ is the probability that player 1 wins times the probabilities that both players cheat.

Let $P(X(\alpha)=d \mid W=1)$ and $P(X(\beta)=d \mid W=2)$ denote the conditional probabilities that agents 1 and 2 are doped if they win. These probabilities are respectively

$$
\begin{aligned}
& P(X(\alpha)=d \mid W=1)=\frac{\alpha(1-\beta)+\sigma \alpha \beta}{(1-\beta)(\sigma(1-\alpha)+\alpha)+\sigma \alpha \beta} \text { and } \\
& P(X(\beta)=d \mid W=2)=\frac{(1-\sigma) \alpha \beta+(1-\alpha) \beta}{(1-\alpha)((1-\sigma)(1-\beta)+\beta)+(1-\sigma) \alpha \beta} .(4)
\end{aligned}
$$

In equilibrium all agents must be indifferent between their strategies, which implies that for the controller and the agents the following conditions must hold.

Controller The controller's expected payoff of testing winner 1 is the conditional probability that winner 1 is doped minus the costs of testing, that is $P(X(\alpha)=d \mid W=1)-k$, while his (expected) payoff of not testing is 0 . In equilibrium, both strategies have to yield the same (expected) outcome, so that the equilibrium condition for testing winner 1 or winner 2 are

$$
\begin{aligned}
& P(X(\alpha)=d \mid W=1)-k=0 \\
& P(X(\beta)=d \mid W=2)-k=0 .
\end{aligned}
$$

Agents The expected payoff for agent 1 of playing $c$ is $E_{1}[c]=\sigma(1-$ $\beta)$ : with probability $(1-\beta)$ player 2 is clean in which case he wins with probability $\sigma$. In all other cases he loses. The expected payoff of playing $d$ is $E_{1}[d]=\left(1-t_{1}\right)(\sigma \beta+(1-\beta))-t_{1}(\sigma \beta+(1-\beta)) P$ : With probability $1-\beta$ player 2 is clean in which case he wins with certainty and with probability $\beta$ player 2 is doped in which case he wins with probability $\sigma$. With probability $\left(1-t_{1}\right)$ he is not tested by the controller in which case he receives the prize 
of value 1 and with probability $t_{1}$ he is tested and receives punishment $P$. Thus the equilibrium condition for agent 1 is

$$
\sigma(1-\beta)=\left(1-t_{1}(1+P)\right)(\sigma \beta+(1-\beta))
$$

The equilibrium condition for agent 2 , which is derived in the same way, is

$$
(1-\sigma)(1-\alpha)=\left(1-t_{2}(1+P)\right)((1-\sigma) \alpha+(1-\alpha))
$$

Lemma 1 In any equilibrium, the favorite player (agent 1) is more likely to dope than the underdog $\left(\alpha \geq \beta\right.$; with strict inequality if $\sigma>\frac{1}{2}$ ). Furthermore, the underdog is more likely to be tested than agent 1 if he wins $\left(t_{2} \geq t_{1}\right.$; with strict inequality if $\left.\sigma>\frac{1}{2}\right)$.

Proof. The fact that in any equilibrium $\alpha \geq \beta$, is a direct consequence of the equilibrium conditions (5) and (6). They require that after winning the conditional probabilities that agent 1 and 2 are doped must be the same. But because agent 2 is more likely to lose if both agents are either doped or clean, he is more likely to be doped if he wins if both agents dope with the same probability (or if he is more likely to dope than agent 1 ). Consequently, $\alpha$ must be greater than $\beta$ in any equilibrium, for otherwise the controller is not indifferent between testing and not testing winner 1 .

The underdog is more often tested because, all else equal, doping is relatively more attractive for agent 2 than playing clean. To see this, let us define the relative attractiveness of doping for agent $i$ as $\frac{E_{i}[d]}{E_{i}[c]}$, which from (7) and (8) is equal to one in any equilibrium. Therefore,

$$
\begin{aligned}
\frac{E_{1}[d]}{E_{1}[c]} & =\left[1-t_{1}(1+P)\right]\left(\frac{\beta}{1-\beta}+\frac{1}{\sigma}\right) \\
& =\left[1-t_{2}(1+P)\right]\left(\frac{\alpha}{1-\alpha}+\frac{1}{1-\sigma}\right)=\frac{E_{2}[d]}{E_{2}[c]} .
\end{aligned}
$$

But since $\left(\frac{\beta}{1-\beta}+\frac{1}{\sigma}\right)<\left(\frac{\alpha}{1-\alpha}+\frac{1}{1-\sigma}\right)$, we have $t_{1}<t_{2}$. That is, because doping is relatively more attractive for the underdog, the underdog must be more likely to be tested if he wins. 
The four equations (5), (6), (7), and (8) describe a system of equations in the four unknowns $t_{1}, t_{2}, \alpha$, and $\beta$. The unique solution is

$$
\alpha^{*}=\frac{1-\Psi-k(1-2 \sigma)}{2 \sigma}, \beta^{*}=\frac{1-\Psi+k(1-2 \sigma)}{2(1-\sigma)}
$$

and

$$
t_{1}^{*}=\frac{\beta^{*}}{k(1+P)}, t_{2}^{*}=\frac{\alpha^{*}}{k(1+P)},
$$

where $\Psi=\sqrt{(1-k)\left(1-k(1-2 \sigma)^{2}\right)}$.

Proposition 2 The strategy profile $\left(t_{1}^{*}, t_{2}^{*} ; \alpha^{*} ; \beta^{*}\right)$ is the unique Bayesian Nash Equilibrium of the control game. In this equilibrium, $P^{*}(W=1)=\sigma$ and $P^{*}(W=2)=1-\sigma$.

\section{Proof. In Appendix.}

In accordance with Lemma 1 the favorite player (agent 1 ) is more likely to be doped than the underdog $\left(\alpha^{*} \geq \beta^{*}\right)$ and is less likely to be tested after winning $\left(t_{2}^{*} \geq t_{1}^{*}\right)$. The favorite's cheating probability is increasing in $\sigma\left(\frac{\partial \alpha^{*}}{\partial \sigma}=\frac{(1-k) \alpha^{*}}{\Psi \sigma}>0\right)$ while the underdog's is decreasing $\left(\frac{\partial \beta^{*}}{\partial \sigma}=-\frac{(1-k) \beta^{*}}{\Psi(1-\sigma)}<\right.$ $0)$. For the testing probabilities we have $\frac{\partial t_{1}^{*}}{\partial \sigma}=-\frac{(1-k) \beta^{*}}{k(1+P) \Psi(1-\sigma)}<0$ and $\frac{\partial t_{2}^{*}}{\partial \sigma}=$ $\frac{(1-k) \alpha^{*}}{k(1+P) \Psi \sigma}>0$. Consequently, the differences in the cheating probabilities $\alpha^{*}-\beta^{*}$ and the testing probabilities $t_{2}^{*}-t_{1}^{*}$ are both increasing in $\sigma$. The agents' cheating probabilities increase in $k$. The effects of $k$ on the testing probabilities are less clear, however, because on the one hand an increase in $k$ makes testing more costly, on the other hand more profitable because the winner is more likely doped. One can show that $\frac{\partial t_{1}^{*}}{\partial k}=\frac{t_{1}^{*}}{k}\left(\frac{\partial \beta^{*}}{\partial k} \frac{k}{\beta^{*}}-1\right)>0$ and $\frac{\partial t_{2}^{*}}{\partial k}=\frac{t_{2}^{*}}{k}\left(\frac{\partial \alpha^{*}}{\partial k} \frac{k}{\alpha^{*}}-1\right)>0$.

Interestingly, the possibility of cheating does not affect the agents' prospects of winning the game. In equilibrium, agent 1 wins with probability $P^{*}(W=1)=\sigma$ and agent 2 with probability $P^{*}(W=2)=1-\sigma$. Thus, the winning probabilities are identical in games, where there are no doping opportunities so that the agents can play only $c$, can choose between $c$ and 
$d$ without controls and punishments (in which case both will play $d$ ) or can choose between $c$ and $d$ with controls and punishments. ${ }^{7}$

Finally, the equilibrium strategies of the agents are independent of the deadweight loss penalty $P$. In contrast, the testing probabilities decrease in $P$. $P$ has no impact on the cheating strategies because all players randomize so as to keep all other players indifferent between their pure strategies. Consequently, increasing $P$ will not affect the cheating probabilities. It will however reduce the testing probabilities because the controller must keep the agents indifferent between cheating and not cheating, and since cheating is less profitable if $P$ increases. The same argument explains also why the agents increase their doping probabilities if $k$ increase, i.e. $\frac{\partial \alpha^{*}}{\partial k}>0$ and $\frac{\partial \beta^{*}}{\partial k}>0$.

\section{The Whistle-Blowing Game}

We now extend the game with a whistle-blowing stage. We model this stage as a signalling game between the controller and the loser, where after the contest, the loser sends a message to the controller. The message space is $\{D, I\}$, where $D$ is the message "The winner is doped" and $I$ is the message "I don't know." Thus, when sending message $D$, the loser "blows the whistle." After receiving a message, the controller decides whether to test the winner. Following a test, the game ends. If he decides not to test, the game ends immediately.

In what follows, we assume that while the equilibrium strategies $\alpha$ and $\beta$ are inferred by (and thus in a sense "known" to) every player in the game, the realizations of the random variables $X(\alpha)$ and $X(\beta)$ are private information of the respective agent. ${ }^{8}$ This private information is used by the

\footnotetext{
${ }^{7}$ Thus, in our game, the fight against cheating cannot be rationalized by fairness arguments. This result relies on our assumption that there are no direct cost of doping. If we modify the game by including (e.g. health) costs of doping $\gamma \geq 0$, the game has an equilibrium in mixed strategies for $\sigma>\gamma>1-\sigma$. The ex ante probability for agent 1 to win in such an equilibrium is $\frac{(\sigma-\gamma)\left(\sigma^{2}+\sigma \gamma-\gamma\right)}{(2 \sigma-1)^{2}} \neq \sigma$ (see Berentsen [4]).

${ }^{8} \mathrm{~A}$ standard assumption in the principal-agent-literature is that the distributions of types (of agents) are commonly known, while the realizations of the random variables
} 
loser to update his beliefs about the winners behavior. In particular, due to the effectiveness of doping, a doped loser can infer with certainty that the winner is doped, which is an inference the controller cannot make.

Our goal is to design an incentive-compatible reward and punishment scheme, which allows the controller to extract the loser's private information about the winner's behavior in the contest. More specifically, we want to design a "whistle-blowing mechanism" between the loser and the controller, where the loser and the controller behave as follows: The doped loser (who infers the winner's behavior with perfect accuracy) "blows the whistle" and the clean loser sends the message "I don't know." The controller tests the winner if and only if the loser blows the whistle.

\subsection{Strategies and Beliefs}

A strategy for the entire game for an agent now consists of three choices; the probability with which he dopes, the signal he sends if he loses and if the realization of the random variable is $c$, and the signal he sends if he loses and if the realization of his random variable is $d$. A strategy for agent 1 is

$$
\delta_{1}=\left(\alpha, m_{1}(c), m_{1}(d)\right)
$$

where $\alpha$ is player 1's doping choice, $m_{1}(c)$ is the probability that player 1 sends message $D$ given that he is clean, and $m_{2}(d)$ is the probability that player 1 sends message $D$ given that he is doped. For example, the strategy $\delta_{1}=(\alpha, 0,1)$ says that agent 1 dopes with probability $\alpha$, sends the message $I$ with certainty if he is a clean loser, and sends message $D$ with certainty if he is a doped loser. Likewise, a strategy for agent 2 is

$$
\delta_{2}=\left(\beta, m_{2}(c), m_{2}(d)\right)
$$

are private information of the agents (see e.g. Laffont and Mortimort [10]). Therefore, our model is an application of a principal-agent model, in which the distributions are determined endogenously as the equilibrium randomization of the competing agents. 
We denote the beliefs of agent 1 after losing the competition if he is clean or doped by ${ }^{9}$

$$
\begin{aligned}
\mu_{1}(X(\beta) \mid X(\alpha)=c) & =\left(\mu_{1}(d \mid c), \mu_{1}(c \mid c)\right) \text { and } \\
\mu_{1}(X(\beta) \mid X(\alpha)=d) & =\left(\mu_{1}(d \mid d), \mu_{1}(c \mid d)\right), \text { respectively. }
\end{aligned}
$$

For example, after losing the competition, $\mu_{1}(d \mid c)$ is agent 1's belief that the winner (agent 2) is doped given that he himself is clean. Note that our assumption on the effectiveness of doping implies that no clean agent will ever win against a doped player. Therefore, $\mu_{1}(c \mid d)=0$ and $\mu_{1}(d \mid d)=1$. Agent 2's beliefs are denoted in the same manner, except that there is a subscript 2 .

The controller's strategy still consists of the testing probabilities $t_{1}$ and $t_{2}$. In contrast to the standard control problem, these testing probabilities are now contingent on the loser's message. Therefore, a (mixed) strategy for the controller is now denoted as $\delta_{3}=\left(t_{1}(I), t_{1}(D) ; t_{2}(I), t_{2}(D)\right)$. His beliefs are denoted by $\mu_{3}($.$) . Thus, \mu_{3}(X(\alpha)=d \mid D)$ denotes the controller's belief that winner 1 is doped if player 2 sends the message $D$. Because there is no confusion possible, we use the more concise notation $\mu_{3}(d \mid W=1, D)$ to denote these same beliefs and $\mu_{3}(d \mid W=2, I)$ is accordingly the controller's beliefs that winner 2 is doped given agent 1's message $I$.

\subsection{Punishments, Rewards, and IC-constraints}

The deadweight loss penalty for doping is still denoted by $P$ and the premium for winning is still 1 . The function $\Phi(s, r)$ specifies the punishments or rewards for the loser from sending message $s \in\{I, D\}$. That is, it describes the payoffs to the loser if he sends the message $s \in\{I, D\}$ and the controller doesn't test $(r=0)$, or the test indicates that winner is clean $(r=C)$, or the test indicates that the winner is doped $(r=D)$. We set $\Phi(s, 0)=0$ for all $s$ because we don't want to punish or reward a loser whose message is "I don't know." Likewise, we set $\Phi(I, r)=0$ for all $r$ because we do not wish

\footnotetext{
${ }^{9}$ The beliefs of the winner are irrelevant for the game so we do not state them.
} 
to reward or punish a loser for any message if there is no test. The payoffs $\Phi(s, r)$ are transfers from the controller to the loser if they are positive and transfers from the loser to the controller if they are negative.

The whistle-blowing signaling stage is depicted in Figure 2. In order to keep the figure simple, we only consider the case when agent 2 is the winner so that the signaling game is between player 1 (the sender) and the controller (the receiver).

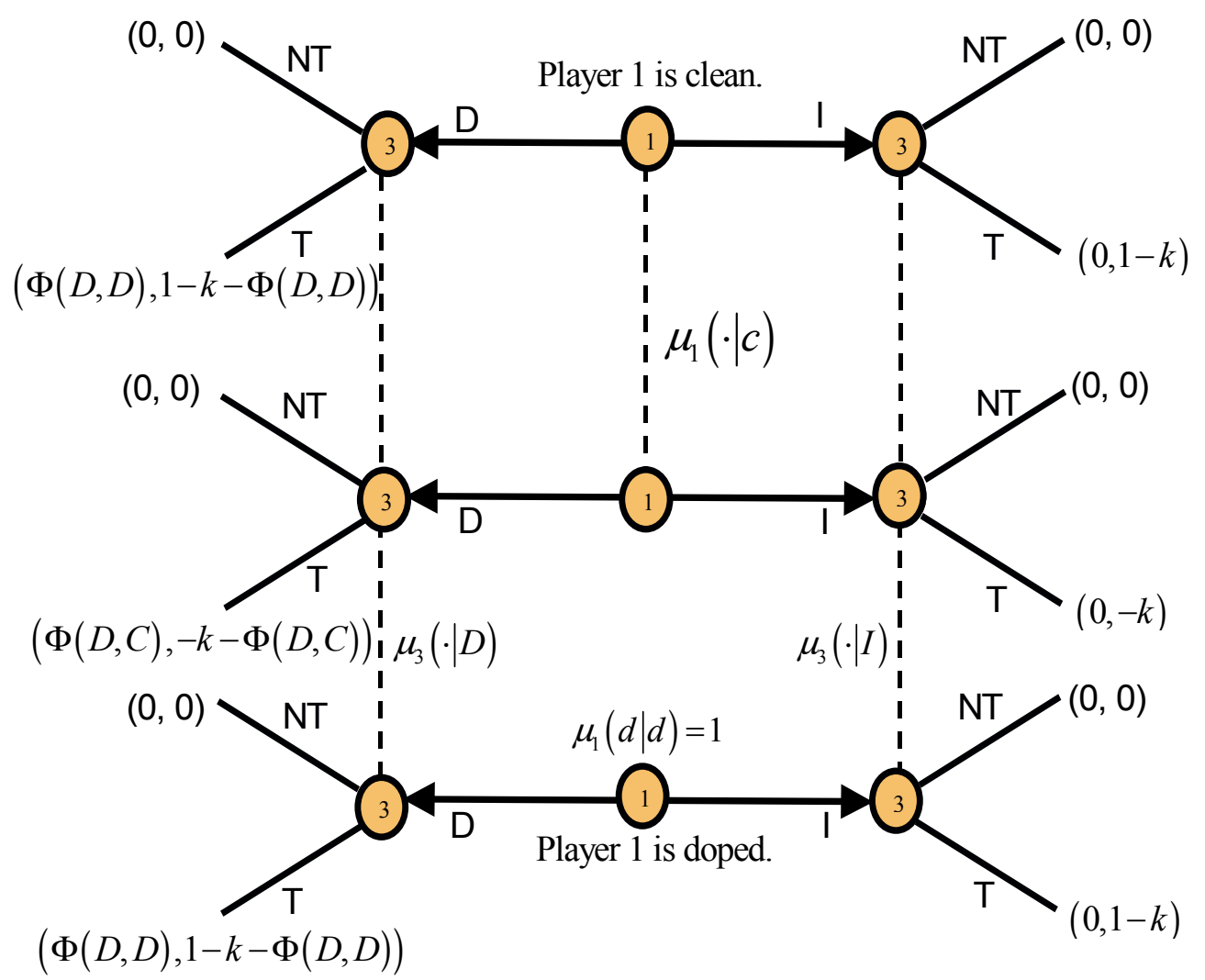

Figure 2. Whistle-blowing signaling stage.

The only non-zero transfers are $\Phi(D, C)$ and $\Phi(D, D)$. We impose the following conditions on them. First, we want a doped loser to send the 
message $D$, which requires that ${ }^{10}$

$$
\Phi(D, D)>0 .
$$

Second, we want a clean loser to send message $I$, which - for reasons that become clear in the proof - requires

$$
-\Phi(D, C)>\frac{\Phi(D, D)}{(1-\sigma) P+\sigma \Phi(D, D)} .
$$

Third, we want the controller to have a dominant strategy to test given message $D$, which requires

$$
1-k>\Phi(D, D) \text { and }-\Phi(D, C)>k \text {. }
$$

Finally, we want the controller not to test if he receives the message $I$, which - for reasons becoming clear in the proof - requires

$$
\Phi(D, D)<\frac{1-\sigma}{\sigma}\left(P-\frac{1-k}{(1-\sigma) k}\right)
$$

\subsection{Whistle-blowing equilibrium}

The solution concept for the game we study is the Perfect Bayesian (Nash) Equilibrium (PBE). A PBE consists of a behavioral strategy profile and a belief structure such that each player's strategy is a best answer to the strategies of all other players, given his beliefs about the history of the game, where these beliefs must be consistent with the equilibrium strategy profile and updated using Bayes' rule (where such is possible).

We first investigate whether the whistle-blowing game has a pooling equilibrium, where the loser sends the same message regardless of whether he is doped or not.

Lemma 3 There are no pooling equilibria, i.e. there are no equilibria in which one agent or both agents send(s) the same message, regardless of whether they are doped or not.

\footnotetext{
${ }^{10}$ Recall that we assume throughout the paper that tests are completely reliable.
} 


\section{Proof. In Appendix.}

We next investigate the existence and uniqueness of a separating equilibrium. For this purpose let us define

$$
\alpha^{* *}=\frac{1}{1-\Phi(D, D)+\frac{1-\sigma}{\sigma} P} \text { and } \beta^{* *}=\frac{1}{1-\Phi(D, D)+\frac{\sigma}{1-\sigma} P} .
$$

Then, we can state the following Proposition.

Proposition 4 The Whistle-blowing game has a unique perfect Bayesian equilibrium with the behavioral strategy profile

$$
\delta_{1}^{*}=\left(\alpha^{* *}, 0,1\right), \delta_{2}^{*}=\left(\beta^{* *}, 0,1\right), \text { and } \delta_{3}^{* *}=(0,1 ; 0,1)
$$

and the belief structure

$$
\begin{array}{cc}
\mu_{1}^{*}(d \mid c)=\frac{\beta^{* *}}{\beta^{* *}+(1-\sigma)\left(1-\beta^{* *}\right)}, & \mu_{2}^{*}(d \mid c)=\frac{\alpha^{* *}}{\alpha^{* *}+\sigma\left(1-\alpha^{* *}\right)} \\
\mu_{3}^{*}(d \mid W=2, I)=\mu_{1}^{*}(d \mid c), & \mu_{3}^{*}(d \mid W=1, I)=\mu_{2}^{*}(d \mid c) \\
\mu_{3}^{*}(d \mid W=2, D)=\mu_{1}^{*}(d \mid d)=\mu_{3}^{*}(d \mid W=1, D)=\mu_{2}^{*}(d \mid d)=1 .
\end{array}
$$

\section{Proof. In Appendix.}

In contrast to the control problem, the equilibrium probabilities of doping now depend negatively on $P$, so that increasing $P$ reduces the frequency of doping. They depend positively on the whistle-blowing reward $\Phi(D, D)$ because an agent obtains this reward only if he is a doped loser and sends the message $D$. Because the controller plays a pure strategy (which is contingent on the message received), the costs of testing $k$ do not affect the agents' equilibrium strategies or beliefs. As in the control game, the favorite player is more likely to be doped than the underdog $\left(\alpha^{* *} \geq \beta^{* *}\right)$, with $\frac{\partial \alpha^{* *}}{\partial \sigma}>0$, $\frac{\partial \beta^{* *}}{\partial \sigma}<0$, and $\frac{\partial\left(\alpha^{* *}-\beta^{* *}\right)}{\partial \sigma}>0$.

Finally, note that the controller tests if and only if both athletes are doped. The frequency of tests is therefore $\alpha^{* *} \beta^{* *}$. 


\section{Comparing the games}

We now compare the doping frequencies, the testing frequencies, and the expected payoffs of the agents and the controller in the whistle-blowing game with the ones in the standard control game.

We first compare the doping frequencies.

Proposition 5 There exist critical values $P_{\alpha} \geq P_{\beta}>0$ defined in the proof such that if $P \geq P_{j}, j^{* *} \leq j^{*}, j=\alpha, \beta$.

\section{Proof. In Appendix.}

According to Proposition (5) if the punishment is sufficiently large, the cheating frequency is lower under the whistle-blowing mechanism than without. Moreover, the more talented player must be punished harsher than the underdog $\left(P_{\alpha} \geq P_{\beta}\right)$ in order to reduce his cheating frequency below the one in the control game. The intuition behind Proposition (5) is that because the deadweight loss penalty $P$ affects the equilibrium behavior of the agents in the whistle-blowing game, increasing $P$ reduces the equilibrium probabilities of doping. This is not the case for the control game, where increasing $P$ will only reduce the equilibrium probabilities of testing. Therefore, if one can freely choose $P$, the equilibrium probabilities both of doping and of testing can be made arbitrarily small under the whistle-blowing mechanism.

We now consider under which conditions the whistle-blowing equilibrium Pareto dominates the equilibrium of the standard control game.

Proposition 6 The unique equilibrium of the whistle-blowing game Paretodominates the unique equilibrium of the standard control game if $P>P_{\alpha}$.

Proof. In the unique equilibrium of the control game, the expected payoff of the controller is zero because in equilibrium he is indifferent between testing and not testing, where not testing yields zero payoff. In contrast, in the whistle-blowing game the controller's expected payoff is strictly positive, because with positive probability he receives the accurate message $D$ and tests the winner, which yields a positive payoff. Consequently, the controller 
is strictly better off in the whistle-blowing game relative to the standard control game.

In both games, the expected payoffs of the agents are equal to the expected payoff of playing clean, which are $\sigma\left(1-\beta^{*}\right)$ and $\sigma\left(1-\beta^{* *}\right)$ for agent 1 and $(1-\sigma)\left(1-\alpha^{*}\right)$ and $(1-\sigma)\left(1-\alpha^{* *}\right)$ for agent 2 , respectively. Thus, in the whistle-blowing game if $\beta^{* *}<\beta^{*}$ agent 1 is strictly better off and if $\alpha^{* *}<\alpha^{*}$ agent 2. Proposition (5) implies that if $P>P_{\alpha}, \beta^{* *}<\beta^{*}$ and $\alpha^{* *}<\alpha^{*}$. Thus, if $P>P_{\alpha}$ the expected utilities of both agents and the controller are strictly larger in the whistle-blowing game than in the standard control game. Consequently, whistle-blowing leads to a strict Pareto improvement.

Proposition 6 makes a strong case for the use of the whistle-blowing mechanism. All participants are ex-ante better off provided that the punishment for behaving illegally can be made sufficiently large $\left(P>P_{\alpha}\right)$.

Finally, we compare the testing frequencies. In the control game the probabilities that player 1 and 2 are tested are $t_{1}^{*} \sigma$ and $t_{2}^{*}(1-\sigma)$, respectively. So the testing frequency is

$$
F_{S C}=t_{1}^{*} \sigma+t_{2}^{*}(1-\sigma)=\frac{\sigma \beta^{*}+(1-\sigma) \alpha^{*}}{k(1+P)} .
$$

Note that $F_{S C}$ strictly increases in $k$, since both $t_{1}^{*}$ and $t_{2}^{*}$ increase in $k$. Hence, $F_{S C}$ is smallest as $k$ approaches 0 .

In the whistle-blowing game, on the other hand, the controller tests if and only if both players are doped, i.e. the testing frequency is

$$
F_{W B}=\alpha^{* *} \beta^{* *}
$$

Note that $F_{W B}$ strictly increases in $\Phi(D, D)$, so that $F_{W B}$ is smallest as $\Phi(D, D)$ approaches 0 and recall that (11) and (14) imply that the whsitleblwoing equilibrium does not exist for $k \leq \frac{1}{1+(1-\sigma) P}$.

Proposition 7 For $P>1, \Phi(D, D)$ can be chosen such that $F_{W B}<F_{S C}$ whenever the whistle-blowing equilibrium exists. 


\section{Proof. In Appendix.}

Proposition 7 makes another strong case for the use of the whistleblowing mechanism, because lower testing frequencies can reduce the cost of implementing controls considerably. Note also that $P>1$ is a sufficient condition, which we have derived under the assumption that the control costs approach zero $(k \rightarrow 0)$, which makes the standard control scheme most effective. For higher values of $k, P$ can be reduced below 1 and we still have $F_{W B}<F_{S C}$.

\section{Conclusions}

In this paper, we have analyzed the role of whistle-blowing in a game with two agents and a controller. We have first derived the equilibrium of a standard control game, where the controller cannot condition his testing strategy on the loser's knowledge. We then compared this (perfect Bayesian) equilibrium to the perfect Bayesian equilibrium of the game in which the loser can "blow the whistle." We have found that whistle-blowing reduces testing frequencies and cheating frequencies. Our whistle-blowing mechanism, therefore, reduces the control cost of the controller and also reduces the likelihood of cheating.

We briefly try to explain why this is so. Note first that in the game without whistle-blowing, cheating frequencies do not depend on the penalty for cheating. In a mixed strategy equilibrium, any player has to make all other players indifferent between the pure strategies they can play. Because we have assumed that penalties are deadweight losses and do no affect the controller's payoff, agents' equilibrium probabilities of cheating are independent of the penalty. Second, in the terms of principal-agent literature, there are two types of winners in our game, those who have cheated and those who have not, and the distribution of these types is determined endogenously by the probabilities with which both agents cheat in the mixed strategies equilibrium. While this distribution can be inferred by every player in the game, the realization of the random variable is private information of the player 
who plays the corresponding mixed strategy. This assumption is completely analogous to the assumption in asymmetric information models that distributions of random variables are public information, while the realizations ("types") are only privately known. But it is also a reasonable assumption, for otherwise, one would have to assume either that the controller also observes this realization (in which case the problem were solved at the outset) or that a player does not observe whether he has cheated or not. Therefore, a loser who has cheated can accuse the winner of having cheated, too, without any risk of false testimony. In equilibrium, the controller controls if and only if the loser blows the whistle. Consequently, the agents determine the frequency of testing themselves through their choice of the probability of doping. In contrast to a standard control game without whistle-blowing, in the whistle-blowing game doping frequencies therefore depend negatively on the penalty for cheating. Increasing the penalty will thus reduce the equilibrium probabilities of cheating in the whistle-blowing game. This is basically why whistle-blowing can reduce both frequencies of testing and frequencies of doping and thus increases welfare relative to the game without whistle-blowing.

Throughout the paper, we have maintained the assumption that cheating is very effective in the sense that a cheater always prevails over a player who does not cheat. We have made this assumption to simplify the analysis, because it enables a loser who has cheated to infer with certainty that the winner has cheated, too. We do not think that the existence of the whistleblowing equilibrium hinges on this assumption. Relaxing this assumption (such that cheating only increases the probability of winning) may, however, have the consequence that other equilibria cannot be excluded. We have also assumed that tests do not involve errors. We suppose that relaxing this assumption would basically have the same consequences as relaxing the assumption that cheating is fully effective.

We think our control problem captures many relevant features of cheating and the fight against cheating. Of course, it does not explicitly capture dynamic issues that are inherent in many cheating situations, because the 
agents can develop new technologies of cheating that allow them to be a step ahead of the controlling authorities, as a consequence of which cheating will never be eliminated. But at the heart of it, the problem of new technologies developed by cheaters is still equally driven by the scarcity of resources disposable for the agency in charge of preventing cheating. For if these resources were not scarce, the agency could do the cheaters' $R \& D$, and the cheaters could not gain even short-term advantages over the agency. Therefore, and because the control problem makes the same prediction, based on the simple, hardly contestable assumption that resources to be devoted to the banning of the illegal activity are limited (see Neue Zürcher Zeitung [12]), the control problem can indeed be seen as capturing the (more) fundamental features of cheating and the fight against it.

\section{Appendix}

Proof of Proposition 1 From (5), (6), (7), and (8), the equilibrium conditions are

$$
\begin{aligned}
\frac{\alpha(1-\beta)+\sigma \alpha \beta}{\sigma(1-\alpha)(1-\beta)+\alpha(1-\beta)+\sigma \alpha \beta} & =k \\
\frac{(1-\alpha) \beta+(1-\sigma) \alpha \beta}{(1-\sigma)(1-\alpha)(1-\beta)+\beta(1-\alpha)+(1-\sigma) \alpha \beta} & =k \\
\left(1-t_{1}(1+P)\right)(\sigma \beta+(1-\beta)) & =\sigma(1-\beta) \\
\left(1-t_{2}(1+P)\right)((1-\sigma) \alpha+(1-\alpha)) & =(1-\sigma)(1-\alpha) .
\end{aligned}
$$

This system of four equations has the unique solution $\left(t_{1}^{*}, t_{2}^{*}, \alpha^{*}, \beta^{*}\right)$. To see that $P^{*}(W=1)=\sigma$, recall that

$$
P^{*}(W=1)=\sigma\left[\left(1-\alpha^{*}\right)\left(1-\beta^{*}\right)+\alpha^{*} \beta^{*}\right]+\alpha^{*}\left(1-\beta^{*}\right)
$$

The first thing to note is that $\left(1-\alpha^{*}\right)\left(1-\beta^{*}\right)=1-k$. Using this information we can simplify (19) to get

$$
P^{*}(W=1)=\sigma(1-k)+\left(k-\beta^{*}\right)\left(\sigma \beta^{*}+1-\beta^{*}\right)\left(1-\beta^{*}\right)^{-1}
$$


Since $t_{1}^{*}=\frac{\beta^{*}}{k(1+P)}$, equilibrium condition (17) implies that

$$
\left(k-\beta^{*}\right)\left(\sigma \beta^{*}+1-\beta^{*}\right)\left(1-\beta^{*}\right)^{-1}=k \sigma,
$$

which implies $P^{*}(W=1)=\sigma$. Equivalent reasoning gives us $P^{*}(W=2)=$ $1-\sigma$.

Proof of Lemma 3 Note first that testing given message $D$ is a dominant strategy for the controller because $-\Phi(D, C)>k$ and $0<\Phi(D, D)<$ $1-k$. Consequently, for a doped loser sending message $I$ is not sequentially rational: The doped loser is sure that the winner is doped and will be tested if he says $D$, so that his expected payoff of saying $D$ is $\Phi(D, D)>0$, while the payoff of saying $I$ is 0 . Hence, sending message $I$ is not sequentially rational for a doped loser.

Therefore, the only candidates for pooling equilibria consist of strategy profiles where the pooling loser always say $(\mathrm{s}) D$. Because testing is a dominant strategy given message $D$, the winner (who may or may not pool himself) will be tested with probability one if he wins. But if an agent is certain to be tested if he wins, he will never dope: If tested with probability one, his expected payoff of doping is negative, while the expected payoff of playing $c$ is positive. But given that his opponent never dopes, the expected payoff of always saying $D$ is negative. Thus, it is not a best response for the pooling to always say $D$. Hence, there are no pooling equilibrium.

Proof of Proposition 4 The proof involves two steps. We first show that the strategy profile and beliefs described in the Proposition (4) is a perfect Bayesian Nash equilibrium. After this we prove that the equilibrium is unique.

Existence: For the controller, the expected payoff of testing given message $I$ has to be negative for both winners. That is, for $i=1,2$

$$
\mu_{3}^{*}(c \mid W=i, I)(-k)+\mu_{3}^{*}(d \mid W=i, I)(1-k)<0,
$$


which implies for $i=1,2$ that

$$
k>\mu_{3}^{*}(d \mid W=i, I) .
$$

In equilibrium $\mu_{3}^{*}(d \mid W=1, I)=\mu_{2}^{*}(d \mid c)=\frac{\alpha^{* *}}{\alpha^{* *}+\sigma\left(1-\alpha^{* *}\right)}$ and $\mu_{3}^{*}(d \mid W=$ $2, I)=\mu_{1}^{*}(d \mid c)=\frac{\beta^{* *}}{\beta^{* *}+(1-\sigma)\left(1-\beta^{* *}\right)}$. Consequently, we need

$$
k>\max \left[\frac{\alpha^{* *}}{\alpha^{* *}+\sigma\left(1-\alpha^{* *}\right)}, \frac{\beta^{* *}}{\beta^{* *}+(1-\sigma)\left(1-\beta^{* *}\right)}\right] .
$$

Inserting the equilibrium values $\alpha^{* *}$ and $\beta^{* *}$ into condition (22) we get

$$
k>\max \left[\frac{1}{1+P-\sigma(P+\Phi(D, D))}, \frac{1}{1+\sigma(P+\Phi(D, D))-\Phi(D, D)}\right]
$$

Note that $1+P-\sigma(P+\Phi(D, D))<1-\Phi(D, D)+\sigma(P+\Phi(D, D))$ because $P+\Phi(D, D)<2 \sigma(P+\Phi(D, D))$ for $\sigma>\frac{1}{2}$. Therefore,

$$
k>\frac{1}{1+P-\sigma(P+\Phi(D, D))}
$$

which can be re-arranged to get condition (14). Note from (13) that the controller has a dominant strategy to test given message $D$. This describes the behavior of the controller.

We next consider the signalling behavior. For a clean agent $i$ message $I$ is sequentially rational if

$$
\mu_{i}^{*}(c \mid c) \Phi(D, C)+\mu_{i}^{*}(d \mid c) \Phi(D, D)<0, i=1,2,
$$

which implies

$$
\begin{gathered}
\left(1-\beta^{* *}\right)(1-\sigma) \Phi(D, C)+\beta^{* *} \Phi(D, D)<0 \text { and } \\
\left(1-\alpha^{* *}\right) \sigma \Phi(D, C)+\alpha^{* *} \Phi(D, D)<0 .
\end{gathered}
$$

Plugging in the values of $\alpha^{* *}$ and $\beta^{* *}$ into (25) and (24) and re-arranging, we get

$$
\begin{aligned}
& -\Phi(D, C)>\frac{\Phi(D, D)}{(1-\sigma) P-\sigma \Phi(D, D)} \text { and } \\
& -\Phi(D, C)>\frac{\Phi(D, D)}{\sigma P-(1-\sigma) \Phi(D, D)} .
\end{aligned}
$$


Because for $P>\Phi(D, D)$ (which is implied by (14)) we have $\sigma P-(1-$ $\sigma) \Phi(D, D)>(1-\sigma) P-\sigma \Phi(D, D)$, the restriction $\frac{1}{(1-\sigma) P-\sigma \Phi(D, D)}>\frac{1}{\sigma P-(1-\sigma) \Phi(D, D)}$ is the stronger one. Thus, we need $-\Phi(D, C)>\frac{\Phi(D, D)}{(1-\sigma) P-\sigma \Phi(D, D)}$, which is condition (12).

For a doped agent $i$ message $D$ is sequentially rational if $\Phi(D, D)>0$ because $\mu_{i}^{*}(d \mid d)=1, i=1,2$. This concludes the signalling behavior.

Finally, we show that $\alpha^{* *}$ and $\beta^{* *}$ are equilibrium strategies. The expected payoff for agent 1 of doping is $(1-\beta)-\sigma \beta P+(1-\sigma) \beta \Phi(D, D)$. In equilibrium, it must be equal to the expected payoff of being clean, which is $\sigma(1-\beta)$. Therefore, the equilibrium probability $\beta^{* *}$ is

$$
\beta^{* *}=\frac{1}{1-\Phi(D, D)+\frac{\sigma}{1-\sigma} P} .
$$

Accordingly, $\alpha^{* *}$ is

$$
\alpha^{* *}=\frac{1}{1-\Phi(D, D)+\frac{1-\sigma}{\sigma} P} .
$$

Note that $\alpha^{* *}>\beta^{* *}$ because $\sigma \geq \frac{1}{2}$.

Therefore, the strategy profile and the beliefs in Proposition 4 constitute a PBE.

Uniqueness: We now show that the equilibrium is unique, provided conditions (11), (12), (13), and (14) hold. From Lemma (3) we know that there are no pooling equilibria. So we focus on separating equilibrium candidates. The plan is as follows: We first show (step 1) that there are no equilibria in which a clean loser sends message $D$ with positive probability. We then show in two steps that there is no equilibrium, in which the controller tests with strictly positive probability given message $I$.

Step 1: We first show that there are no equilibria in which $m_{i}(c)>0$ for at least one agent $i$. To see this, note that $m_{i}(c)>0$ implies that

$$
\mu_{i}(d \mid c) \Phi(D, D)+\mu_{i}(c \mid c) \Phi(D, C) \geq 0
$$

$i=1,2$. But if (30) holds, then

$$
\mu_{i}(d \mid c)(1-k)+\mu_{i}(c \mid c)(-k)>0
$$


because in equilibrium $\mu_{3}^{*}(d \mid W=j, I)=\mu_{i}^{*}(d \mid c)$ and $\mu_{3}^{*}(d \mid W=j, D)=$ $\mu_{i}^{*}(d \mid d), j \neq i$. Hence, if the expected payoff of clean agent $i$ of sending message $D$ is non-negative, then the controller's expected payoff of testing given message $I$ is strictly positive. But this implies that winner $j, j \neq i$, is tested with certainty. Consequently, he will never dope, which contradicts (30). Thus in any equilibrium $m_{i}(c)=0, i=1,2$.

Step 2: We now show that there is no separating PBE, in which the controller's testing probabilities are $0<t_{i}(I) \leq 1$ and $0<t_{j}(I) \leq 1, i, j=$ 1,2 .

First, consider the case with $t_{i}(I)=1$ for at least one $i$. Given that sequential rationality requires the doped loser to say $D$ and that testing is a dominant strategy given message $D\left(t_{i}(D)=1, i=1,2\right), t_{i}(I)=1$ implies that agent $i$ will always be tested, which cannot be an equilibrium.

Next consider the case with $0<t_{i}(I)<1$ for $i=1,2$. This cannot be an equilibrium. For such a strategy profile to be an equilibrium, the controller's expected payoff of testing the winner given that the loser has sent message $I$ must be equal to the payoff of not testing, which is zero. Thus, for the controller we need

$$
\frac{\alpha}{\sigma(1-\alpha)+\alpha}-k=0 \text { and } \frac{\beta}{(1-\sigma)(1-\beta)+\beta}-k=0
$$

yielding $\widehat{\alpha}=\frac{k \sigma}{1-k(1-\sigma)}$ and $\widehat{\beta}=\frac{k(1-\sigma)}{1-k \sigma}$.

The payoff to the loser if the winner is tested positive is $\Phi(D, D)$. Because agents 1 and 2 must be indifferent between the two (pure) behavioral strategies available, we need

$$
\begin{aligned}
& (1-\beta)\left(1-t_{1}(1+P)\right)-\sigma \beta P+(1-\sigma) \beta \Phi(D, D)=\sigma(1-\beta) \text { and }(32) \\
& (1-\alpha)\left(1-t_{2}(1+P)\right)-(1-\sigma) \alpha P+\sigma \alpha \Phi(D, D)=(1-\sigma)(1-\alpha \gamma 33)
\end{aligned}
$$

Using $\widehat{\alpha}$ and $\widehat{\beta}$ and re-arranging yields

$$
\begin{aligned}
t_{1} & =\frac{1-\sigma}{(-1+k)(1+P)}(-1+k-k \Phi(D, D)+k \sigma(\Phi(D, D)+P)) \\
t_{2} & =\frac{\sigma}{(1-k)(1+P)}(-1+k(1+P-\sigma(\Phi(D, D)+P)))
\end{aligned}
$$


By assumption $t_{1}$ and $t_{2}$ are strictly positive probabilities. Because the fraction in equation (34) is negative, the term in brackets in equation (34) has also to be negative. Thus we must have $k-k \Phi(D, D)+\sigma k(\Phi(D, D)+P)<1$. On the other hand, because the fraction in (35) is positive, the term in brackets needs to be greater than zero. Thus, $k+k P-\sigma k(B+P)>1$. But this can never simultaneously be the case, because

$$
k-k \Phi(D, D)+\sigma k(\Phi(D, D)+P) \geq k+k P-\sigma k(\Phi(D, D)+P)
$$

implying

$$
2 \sigma k(\Phi(D, D)+P) \geq k(\Phi(D, D)+P),
$$

which always holds for $\sigma \geq \frac{1}{2}$, contradicting the assumption that $t_{1}$ and $t_{2}$ are strictly positive probabilities.

Step 3. There is no equilibrium with $t_{i}(I)=0$ and $0<t_{j}(I)<1$ for $j \neq i$. Assume to the contrary $t_{2}(I)=0$ and $0<t_{1}(I)<1$. Then, agent 1 must keep the controller indifferent. That is, $\alpha$ is such that

$$
\frac{\alpha}{\alpha+\sigma(1-\alpha)}-k=0
$$

On the other hand, agent 1 must also keep agent 2 indifferent. Hence, $\alpha$ also solves

$$
(1-\alpha)+\sigma \alpha \Phi(D, D)-(1-\sigma) \alpha P=(1-\alpha)(1-\sigma) .
$$

But $\alpha$ solves (38) and (39) if and only if $\Phi(D, D)=\frac{1-\sigma}{\sigma}\left(P-\frac{1-k}{k(1-\sigma)}\right)$, which contradicts (14). Hence, this is not an equilibrium. Analogous reasoning applies in the converse case with $t_{1}(I)=0$ and $0<t_{2}(I)<1$.

This concludes the proof that the equilibrium is unique.

Proof of Proposition 5. The proof is straightforward and only involves the comparison of $\alpha^{* *}$ with $\alpha^{*}$ and $\beta^{* *}$ with $\beta^{*}$. The critical values are

$$
\begin{aligned}
& P_{\alpha}=\frac{\sigma}{1-\sigma}\left(\frac{1}{\alpha^{*}}-1+\Phi(D, D)\right) \text { and } \\
& P_{\beta}=\frac{1-\sigma}{\sigma}\left(\frac{1}{\beta^{*}}-1+\Phi(D, D)\right), \text { respectively. }
\end{aligned}
$$


To show that $P_{\alpha} \geq P_{\beta}$, note that $\frac{\sigma}{1-\sigma}\left(\frac{1}{\alpha^{*}}-1\right) \geq \frac{1-\sigma}{\sigma}\left(\frac{1}{\beta^{*}}-1\right)>0 \Longrightarrow$ $P_{\alpha} \geq P_{\beta}$. Hence, if

$$
\frac{\sigma^{2}}{(1-\sigma)^{2}} \geq\left(\frac{1-\beta^{*}}{\beta^{*}}\right)\left(\frac{\alpha^{*}}{1-\alpha^{*}}\right)
$$

$P_{\alpha} \geq P_{\beta}$. Equations (5) and (6) imply that $\frac{1-\sigma}{\sigma}=\left(\frac{\beta^{*}}{1-\beta^{*}}\right)\left(\frac{1-\alpha^{*}}{\alpha^{*}}\right)$. Multiplying both sides by this term and re-arranging, we get $\sigma \geq \frac{1}{2}$.

Proof of Proposition 7. We show that the limit of $F_{S C}$ as $k$ goes to 0 is greater than the limit of $F_{W B}$ as $\Phi(D, D)$ approaches 0 (where the whistle-blowing equilibrium does not necessarily exist). Because $F_{S C}$ strictly increases in $k$ and $F_{W B}$ is independent of it, $F_{S C}>F_{W B}$ for any greater $k$ then follows immediately. Therefore, $F_{S C}>F_{W B}$ will also be feasible through appropriate (i.e. sufficiently small) choice of $\Phi(D, D)$ for any $k$ for which the whistle-blowing equilibrium exists. The limit

$$
\lim _{\phi \rightarrow 0} F_{w b}=\frac{\sigma(1-\sigma)}{(1-\sigma+\sigma P)(\sigma+P-\sigma P)}
$$

is straightforward to find.

Using L'Hôpital's rule, we can determine the limit of $F_{S C}$, which is

$$
\lim _{k \rightarrow 0} F_{s c}=2 \frac{\sigma(1-\sigma)}{1+P} .
$$

Now, for $P>1$ we have

$$
2 \frac{\sigma(1-\sigma)}{1+P}>\frac{\sigma(1-\sigma)}{(1-\sigma+\sigma P)(\sigma+P-\sigma P)}
$$

since inequality (40) can be simplified to yield

$$
(1-P)^{2} \sigma(1-\sigma)>\frac{1}{2}(1-P)
$$

which is always true because for $P>1$, the right-hand side is negative, while the left-hand side is positive. 


\section{References}

[1] Aumann, Robert J. and Sergiu Hart (editors), Handbook of Game Theory, Vol. 3, Elsevier, Amsterdam, 2002.

[2] Avenhaus, Rudolf (1997), Entscheidungstheoretische Analyse der Fahrgast-Kontrollen, Der Nahverkehr 9: 27-30.

[3] Avenhaus, Rudolf, Von Stengel, Bernhard and Shmuel Zamir (2002), Inspection Games, in: Handbook of Game Theory, Vol. 3, edited by Robert J. Aumann and Sergiu Hart.

[4] Berentsen, Aleksander (2002), The Economics of Doping, European Journal of Political Economy, Vol. 18, Issue 1, pp. 109-127.

[5] Dixit, Avinash (1987), Strategic Behavior in Contests, American Economic Review, Vol. 77(5): 891-8.

[6] Economist (2003), Christine Casey: whistleblower, Jan 16th.

[7] Gibbons, Robert, A Primer in Game Theory, Prentice Hall, New York, 1992.

[8] Greenberg, Joseph (1984), Avoiding Tax Avoidance: A (Repeated) Game-Theoretic Approach, Jounral of Economic Theory Vol. 32:1-13.

[9] Güth, Werner and Rüdiger Pethig (1992), Illegal Pollution and Monitoring of Unknown Quality, Chapter 10 in Petrig (ed.) (1992).

[10] Laffont, Jean-Jacques and David Martimort, The Theory of Incentives : the Principal-agent Model, Princeton University Press, Princeton, 2002 .

[11] Lazear, Edward P. and Sherwin Rosen (1981), Rank-Order Tournaments as Optimum Labor Contracts, Journal of Political Economy Vol. 89(5): 841-64. 
[12] Neue Zürcher Zeitung (2003), Kampf gegen Windmühlen, February 27 (Nr. 48): 47.

[13] Pethig, Rüdiger (editor), Conflicts and Cooperation in Managing Environmental Resources, Springer, Berlin, 1992. 